\title{
Vehicle to Vehicle communications applied to Road Intersection Safety, Field Results
}

\author{
Javier Ibanez-Guzman, Member, IEEE, Stephanie Lefevre, Abdelkader Mokkadem and \\ Sylvain Rodhaim
}

\begin{abstract}
Road intersections represent one of the most complex configurations encountered when traversing road networks. A high percentage of accidents occur at these locations. The introduction of wireless communications technologies onboard passenger vehicles is enabling the sharing of information and through it enhancing the situational awareness of vehicle drivers. In this paper the implementation of safety applications for cooperative vehicle systems is presented applied to road intersection safety. The system relies on three fundamental technologies: communications, localization and the modelling of the environment surrounding the subject vehicle. The paper centres in a case study, the priority crossing of an Emergency Service Vehicle at an intersection. An analysis of the issues identified during the implementation and testing is included. The implementation represents an instance of the architecture developed for cooperative vehicles applications as part of the European project, SAFESPOT.
\end{abstract}

\section{INTRODUCTION}

$\mathrm{A}$ $\mathrm{N}$ intersection is defined as a road junction where two or more roads meet, cross or split at the same level. Between the years 1996-2004, almost 61.000 persons were killed in traffic accidents at intersections, in 14 European Union countries. This represents $21 \%$ of all traffic accident fatalities [1]. In 2004, out of 1.2 million injury accidents in the Europe of 27, $43 \%$ occurred at intersections. These were responsible for 10000 fatalities or $21 \%$ of deaths in road accidents [2]. The situation is more alarming in the newly industrialised countries where accidents are a societal and economic problem.

Changes in legislation combined with an increased use of onboard safety systems have reduced the number of fatalities within the past years e.g. the number of fatalities at intersections for the years 1996-2004 has been reduced by $21.2 \%$ [1]; nevertheless numbers remain high. Current vehicles incorporate a combination of passive and active safety systems designed to protect vehicle occupants. Passive systems include safety belts, air-bags, etc. they function once a collision occurs. Active safety systems are designed to prevent accidents or to reduce the speed at which collisions occur. These include lane departure systems, pre-crash

J. Ibanez-Guzman, S. Lefevre and A. Mokaddem are with Renault S.A., 1 Av. du Golf, 78288 Guyancourt, France (e-mail: javier.ibanez-guzman | stephanie.lefevre | abdelkader.mokaddem@ renault.fr).

S. Rodhain, is with Viveris Technologies, Parc d'Affaires SILIC, 1, rue Traversière, 94598 Rungis, France. braking, etc. They use exteroceptive sensors to identify safety risks and either inform the driver or act on the vehicle. These systems are a major progress in safety. However, the physics involved in traffic situations, costs and complexity limit their effectiveness.

Vehicle motion in traffic environments is a "SpatioTemporal" problem, if the spatial evolution of the vehicles is known within a time sequence, by projecting it into a digital representation of the environment; it is possible to build a world model. The availability of low cost wireless communications systems onboard passenger vehicles should permit the sharing of information in extended areas. The world model could provide an enlarged situational view of the environment well beyond the limitations of onboard exteroceptive sensors. This results in an extended awareness for the driver and safety systems. That is, an early understanding of potential risks could be attained allowing the anticipated deployment of safety measures. Within this perspective the European Commission (EC) has allocated in 2008 an exclusive frequency for vehicle to vehicle (V2V) and vehicle to infrastructure communications (V2I) or V2X, to be used for safety and traffic information flow. This comprises $30 \mathrm{MHz}$ of spectrum in the $5.9 \mathrm{GHz}$ band [3].

This paper presents the implementation of use cases related to intersection safety using $\mathrm{V} 2 \mathrm{~V}$ wireless communications. The partial results of the co-funded project SAFESPOT (Cooperative Systems for Road Safety), by the EC Information Society and Media. SAFESPOT is an integrated project under their $6^{\text {th }}$ Framework Programme; it comprises major vehicle OEMs, rank 1 automotive suppliers and leading universities/laboratories across Europe [4].

The paper is organized as follows: Section II summarises the accidentology analysis made to define the context where intersection accidents occur and to identify design constraints. A formulation of the road intersection problem and associated use cases is made in Section III. A system description of the architecture and related technologies is presented in Section IV. The implementation and results of the field trials are included in Section V. Finally, Section VI concludes the paper with emphasis on potential research problems that the implementation has identified.

The paper centres on the implementation of the system, a thorough literature review was made as part of the SAFESPOT project, it can be found in [5]. 


\section{ANALYSIS OF INTERSECTION SAFETY}

Accidents across Europe have been reduced over the past years, though figures remain high. In the Europe of 27 (2004): $43 \%$ of injury related accidents, $21 \%$ of fatalities, $44 \%$ of victims and $34 \%$ of the seriously injured out of the total number of accidents were at road intersections [2].

Intersection accidents occurred mainly in rural areas $(80 \%)$, they have a low severity. Fatalities at junctions mostly occur inside urban areas (42\%). Of injury related accidents at intersections, $23 \%$ involved at least one passenger car and resulted in $10 \%$ of all fatalities. In the United Kingdom (2004), more than one third of the overall road accident fatalities occurred at junctions ( $35 \%)$, whereas in Greece fatalities at junctions constitute a minority [1].

Lately, the number of fatalities at junction inside urban areas has been decreasing more quickly than those occurring in rural areas. This is mainly due to changes in attitude, compliance with the law and better road design.

\section{A. Understanding Intersection Accidents}

An intersection is a junction that contains a crossing or a connection of two or more roadways not classified as a driveway access. A junction is a location where traffic can change between different routes, directions, or sometimes modes of travel. The former includes T-junctions, Yjunctions, crossroads and level crossings, where as the later comprise exit junctions.

An intersection-related crash occurs when the first harmful event happens outside but near a road intersection and involves a vehicle which was engaged or should have been engaged in making an intersection-related manoeuvre such as a turn. When an accident occurs, a dysfunction exists that could be associated either to the vehicle, the driver or infrastructure. It could involve one or two vehicles, a vehicle and vulnerable road users, a vehicle and two wheeled vehicles, etc. Is there an age distribution? At what time of the day accidents occur? Was the weather a factor? What were the trajectories of the vehicles? These are important considerations that direct the design of the road intersection safety system.
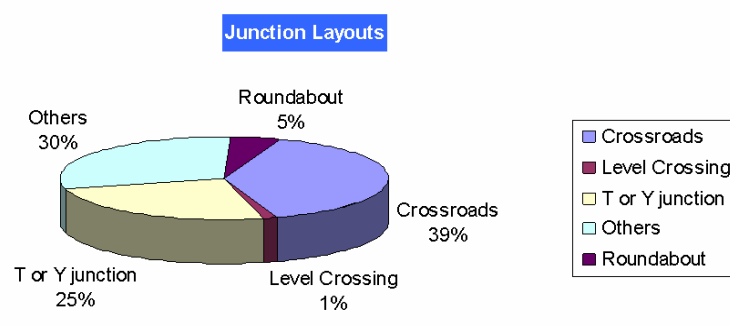

Fig. 1. Distribution of Intersections accidents by road geometry

Road Geometry. Is there a type of road geometry where most accidents at intersections occur? Figure 1 shows the distribution of road accidents at intersections with respect to the road geometry [2]. Most accidents occur at crossroads, followed by those at $\mathrm{T}$ and $\mathrm{Y}$ junctions, roundabouts and level crossings.

Accidents at crossroads involve "cutting scenarios", that is the path of the converging vehicles will result in a contact point, independent of the type of signals or traffic lights. The vehicles arrive perpendicular to each other or when a turning manoeuvre is performed by any of the drivers involved.

For analysis purposes, the Subject Vehicle (SV) is the one observed or where the safety system will be hosted, whilst an Intruder Vehicle (IV) is the one likely to collide with the SV. Figure 2 represents the trajectories and points of contact that occur most frequently between two vehicles at a crossroads.

Statistics show that accidents most likely occur for scenarios 1.1 and 1.2, as shown in Figures 2a) and $2 \mathrm{~b}$ ) with 29 and $27 \%$ of accidents resulting in persons killed or seriously injured out of all the total fatalities at intersections. Accidents involving rear collisions at intersection represent $5 \%$. Safety Systems should operate mainly in this type of intersections. Accidents in "cutting scenarios" represent for the elderly $37 \%$ of fatalities and $60 \%$ of injuries. For persons older than 80 years, half of fatal crashes occur at intersections.

Road and Traffic Signals. The distribution varies very much across Europe. In France, intersections without any control experience few accidents (10\%), by contrast in Spain these are $30 \%$. In the UK, accidents at intersections with "give way" signs represent $~ 58 \%$ (most accidents) whilst in France less than $10 \%$. Accidents at traffic lights vary between $20 \%$ in the UK and $~ 60 \%$ in France.

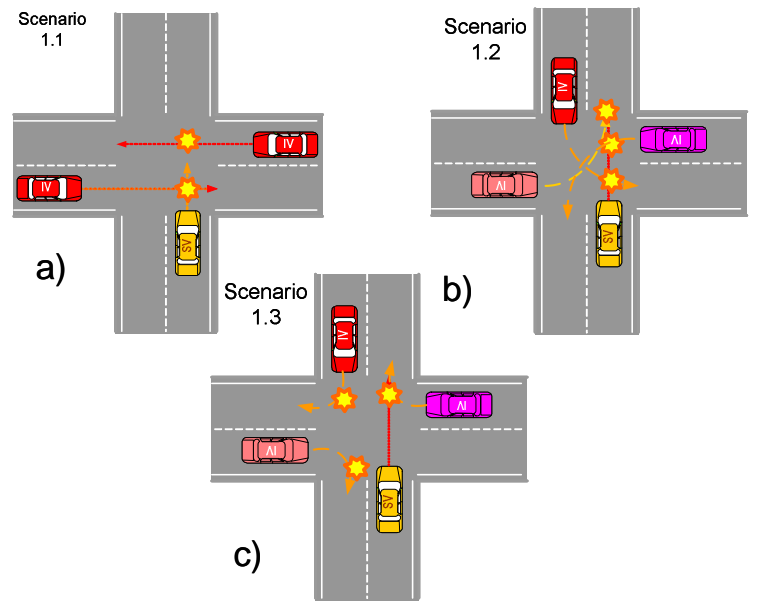

Fig. 2. Vehicle trajectories for "cutting scenarios".

Road and Traffic Signals. The distribution of accidents at intersections having road and traffic signals varies very much across Europe. In France, intersections without any control experience few accidents (10\%), by contrast in Spain these are $30 \%$. In the UK, accidents at intersections with "give way" signs represent $~ 58 \%$ (most accidents) whilst in France less than $10 \%$. Accidents at traffic lights vary between $20 \%$ in the UK and $\sim 60 \%$ in France.

Environmental Conditions. Contrary to common belief accidents mainly occur at daylight or twilight when good 
visibility conditions exist (48\%), those at night represent $23 \%$. Similarly, accidents at intersections occur when conditions are dry (between 82 to $90 \%$ ).

Involved Actors. In most cases two vehicles (67 to $82 \%$ ). One pedestrian and one vehicle ( $9 \%$ to 14\%). Accidents involve mainly passenger cars ( 85 to $90 \%$ ), followed by powered two wheelers and pedestrians.

Driving Situation. Several phases exist, as a vehicle arrives to an intersection, normal, crossing, turning, etc. 59 to $67 \%$ of all intersection situations occurred while the driving phase was normal (no manoeuvre), with 9 to $18 \%$ when drivers turned left.

Collision Causes. It is estimated that in normal conditions, $43 \%$ were driver inattention, followed by $12 \%$ as a deliberate violation of the traffic signal. When the vehicle is making a manoeuvre: $70 \%$ will be driver inattention (did not observe the incoming vehicle) and $16 \%$ a deliberate manoeuvre [6].

\section{INTERSECTION SAFETY COOPERATIVE SYSTEM}

Communications between vehicles and infrastructure provide a network that allows for the dynamic sharing of information amongst entities in the road network. That is, information acquired by the vehicles or infrastructure via their own sensors can be shared with other vehicles or with another part of the infrastructure. Figure 3 represents a crossroad where different types of vehicles converge. The subject vehicle (SV) arrives to the intersection, the driver needs to be aware of the presence of all vehicles or vulnerable road users that represent a risk. Although use of perception systems should enhance driver awareness, as represented by the triangular shape in the figure, occlusion will mask several potential risks e.g. the distracting vehicle (DV), the power two wheeled vehicle (PTW), etc. Further, if there is an Intrusion Vehicle (IV) arriving at a prohibited speed, for the driver of the SV will be difficult to know that the IV will be travelling too fast to brake at the stop line on time. When the IV enters the SV sensor field of view, it will be likely too close already.

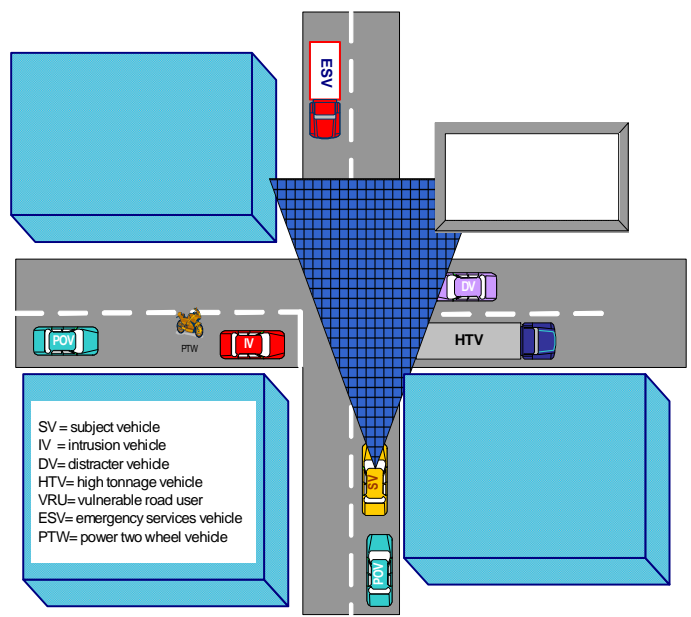

Fig. 3. Configuration of a crossroad intersection in an Urban
However, if each vehicle can transmit their position, speed and other data, by associating this information onto a digital map representing the road geometry and other contextual information an extended digital representation of the vehicle immediate environment could be built as shown in Figure 4. Thus an application running in the SV can analyse and identify the possible risks informing the driver beyond what current sensors could provide. The figure shows the risk vehicles in red. By knowing the speed at which they are evolving, their distance to the intersection at the time of the query plus the state of the SV, it is possible to warn or even act in the SV. This is the Safety Margin concept deployed in the SAFESPOT project.

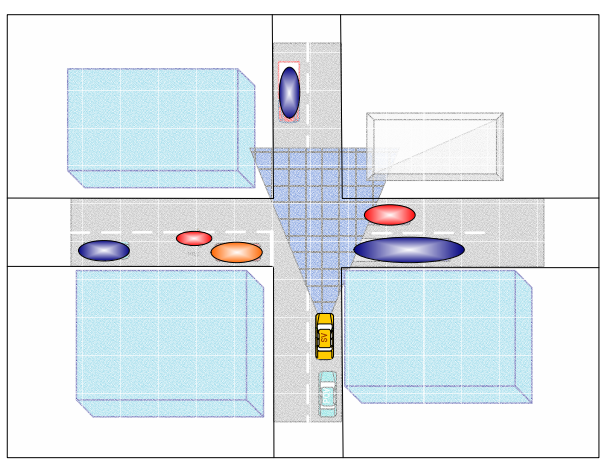

Fig. 4 Perception from the SV perspective using V2V communications (after [7])

Thus by sharing vehicle state information, projecting it on the road geometry, it is possible to extend the driver situational awareness. The fundamental functions for a V2V safety system would consist of a Wireless Communications dynamic network, a Digital Map and a Localisation system.

\section{A. Road Intersection, Safety Use Cases}

The intersection safety problem is confined to Use Cases representing different instances of safety risk that can be addressed by cooperative vehicles. In SAFESPOT, these were described thoroughly [4]. Some of the most representative use cases are: Crossing a road intersection independent of the right of way, Assisting a vehicle to make a left hand turn, Turning left at an intersection whilst having an occluded view, Building a right of way for an Emergency Vehicle approaching an intersection, etc.

\section{SYSTEM LEVEL DESCRIPTION}

The design principles applied centre on the depiction of the SV ego-state and information received from other vehicles within its immediate environment on a digital representation of the road geometry. That is, a hierarchical representation of the environment where all the information will be mapped and associated with respect to time. It requires a multi-level representation having multi-resolutions and different sizes. Each level would contain static and dynamic data including the position of the hosting vehicle (ego-position) and the geo-localised projections of the vehicle states from data received via the wireless network. 
The principle is similar to the 4-D/RCS architecture for autonomous unmanned ground vehicles formulated by $\mathrm{J}$. Albus [8].

The map structure in SAFESPOT is known as the Local Dynamic Map (LDM). In the automotive world digital maps representing road geometries and associated features are used intensely in car navigation systems. These are used as the basis for the LDM. The main map suppliers TeleAtlas and Navteq are involved in the project. Separate implementations of the LDM were made; these were associated to different sites. The implementation used the Navteq version. A description of one instance of the LDM can be found in [9].

The relative position of the vehicles is another primary function not only the $S V$ position has to be projected onto the LDM using map-matching techniques but also the positions of other vehicles received via the wireless network [7]. The use cases implemented are vehicle position dependent with the spatial association requiring temporal information. This is difficult as position estimates might be at different rates with processing and transmission delays likely to occur. Within SAFESPOT different solutions to the positioning problem were studied, including the use of landmarks, dead reckoning, dGPS, etc.

As vehicles enter within the communications range of their onboard equipment, they form dynamic ad-how networks for information exchange. These use the $5.9 \mathrm{GHz}$ frequency based on the IEEE 802.11p. They allow for Multihop forwarding and geo-routing and accessibility with high priority for the exchange of critical information.

The information from the SV and in the incoming messages needs to be fused and interpreted. This is done as part of the Situation Refinement in the data fusion process. The SV position information is projected onto the LDM. The position of the other vehicles embedded in the incoming message is projected in time to compensate for latencies in the system, a temporal alignment. The compensation was made via a path-prediction method, in which the vehicle position, speed and heading will be projected in time as an estimate. This projection is made to the time at which the SV ego-state is used.

Figure 5 shows the System Architecture. The major components are the LDM, the Positioning System, VANET router/Message Generation, Data Fusion and the Application software. The later implements the use cases by generating the messages associated to the Safety Margin. The Data Fusion unit performs a very important task. It maintains the coherence of the LDM via a set of functions: Situation Refinement, Spatial and Temporal Alignment. The only system component writing onto the LDM is the output of the data fusion process. The cooperative applications are triggered following events detected in the LDM. These in turn will effect some queries on the LDM to decide whether or not to inform the SV driver. The SV ego-state is generated by combining information from the vehicle proprioceptive sensors with that from the positioning system. Time stamping the information is very important for this purposes all computers clocks are synchronised using the GPS time issued from the positioning system.

Similar systems have been studied; these are in response to specific situations. A general review in this area can be found in [11] and [12].

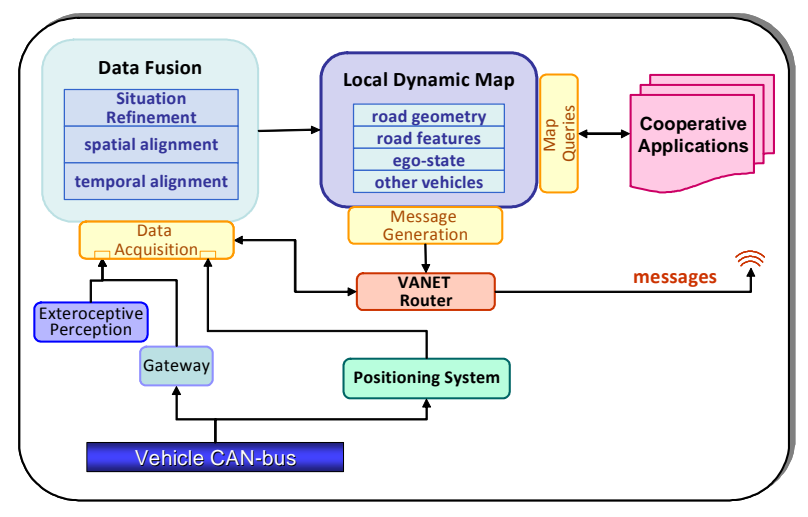

Fig. 5. SAFESPOT System Architecture

\section{SYSTEM IMPLEMENTATION AND APPLICATION}

The software was developed by several project partners. The final system consisted of several PC-type computers running Windows XP and Linux OS. All computers were linked using an Ethernet switch. To decouple the positioning problem and to centre on the project applications, the system used an advanced positioning system that consisted of a loosely coupled GPS, odometry and an Inertial Measurement Unit. The resulting accuracy was less than a metre which makes the projection of the vehicles' positions onto the LDM sufficient for the implemented applications. The systems were integrated onboard two Renault vehicles, an Espace IV and a Laguna III. A schematic representation of the implemented system is shown in Figure 6. The vehicles were modified to supply sufficient electrical power and a gateway to the CAN-bus.

The system implementation required enriched digital. For this purpose special maps were created based on the road geometries found in existing navigation systems.

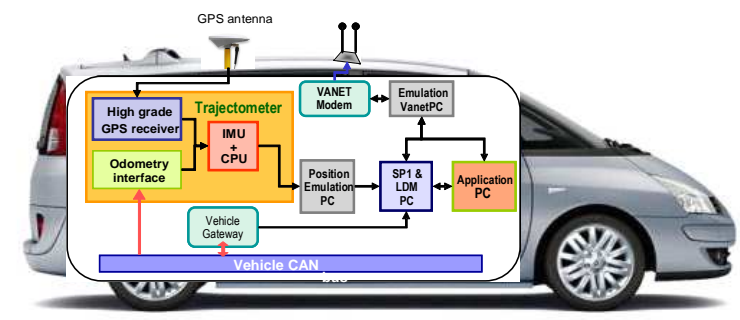

Fig. 6 Schematic representation of the implemented system

\section{A. Use Case. Arrival of an Emergency Vehicle to an Intersection}

For testing purposes the priority crossing of an 
intersection by an Emergency Service Vehicle (ESV) was chosen. The rationale is two fold: First, it enables the testing of the SAFESPOT system on a simple case; priority is given to the ESV and thus no complex decision process is involved. Second, in the event of deployment, if all ESVs and related services are equipped with communications systems, vehicles equipped with V2X systems shall benefit directly from the technology.

Scenario. An ESV broadcasts its presence (at $2 \mathrm{~Hz}$ ) as it approaches an intersection indicating its position and expected trajectory. The vehicles in the neighbourhood will receive this information, those close to the intersection and converging to it will be considered as relevant. Vehicles travelling perpendicular to the ESV will estimate their distance to the next intersection, and after time alignment the distance of the ESV to the same intersection will be calculated. An evaluation of the risk is made based on the vehicles speeds and distances to the intersection, plus an estimation of the distance needed for them to brake [7]. According to this ratio, three levels of warnings can be shown to the driver (yellow, orange, red).

If the ESV travels along the same road as the SV vehicle, its presence is notified to the driver. The intensity of the warning depends on the inter-distance between vehicles. Figure 7 illustrates the concept and shows an instance of the HMI used.

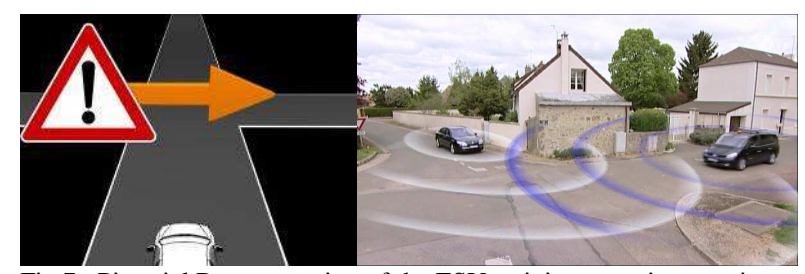

Fig 7. Pictorial Representation of the ESV arriving to an intersection. Warning shown to the SV driver (the saloon car)

Trials were conducted at La Brosse area and at the Satory test track (Guyancourt, France). The first is next to a village whilst the second is a vehicle test site. Vehicles were run at speeds between 20 and $70 \mathrm{Km} / \mathrm{h}$, the upper limit being a safety constraint. Warning signs will appear on a video screen, as shown in Figure 7 when the application was triggered.

A total of four use cases were tested: An accident at an Intersection, one of the vehicles pretends to have an accident while another vehicle approaches the intersection. Obstructed view at intersection, in this case the vehicle obstructing the line of sight was a non SAFESPOT vehicle. Permission Denial to go ahead, one vehicle is standing at the intersection while the other one is approaching. Only visual warnings are given. The most complex use case relates to the Approaching Emergency Vehicle Warning. It uses the full SAFESPOT architecture and V2V communications with both vehicles in motion. The later was demonstrated at the Satory track site where vehicles were driven by people unfamiliar with the technology. Whilst no quantitative evaluations were made, nearly 400 passengers were shown the application (InterTraffic 2010 exhibition, Amsterdam). The application was well perceived.

\section{B. Implementation and Functional Observations}

The system application was written in $\mathrm{C}++$ and was hosted in the SAFESPOT software architecture. The following observations were made:

The first relates to limits of the $802.11 \mathrm{p}$ as an important barrier to situational awareness. The frequencies at which the communication devices operate $(5.9 \mathrm{GHz})$ make them sensitive to obstacles. The vehicles communicate when they are within line of sight of each other. When this occurred, communications could be established up to $700 \mathrm{~m}$ with packet loss fewer than $8 \%$. However, a thin wall of trees obstructing the line of sight will break communications even at $50 \mathrm{~m}$ (25\% packet loss). Better performances could be achieved with better antenna layouts or different modems. Other test included the use of four vehicles at speeds up to $70 \mathrm{Km} / \mathrm{h}$ without a perceptible reduction in performance. During the InterTraffic demonstration, up to 20 nodes were in operation within a confined space, there was not performance reduction.

The second observation is map-matching algorithm used to localize the vehicle on the LDM cannot not always be applied successfully to localize other vehicles on the LDM (from data received via V2X). The reason is that information held by the SV about another vehicle (known as the Principal Other Vehicle - POV) is not always sufficient. As a result the system onboard the SV will not be aware that the two vehicles are heading towards the same intersection, and hazardous conditions might arise. Figure 8 illustrates what occurred during one of the field experiments. Initially, the $\mathrm{SV}$ and POV are $\sim 500 \mathrm{~m}$ apart, with both vehicles establishing a communications link. The vehicles drive towards the same intersection, without any communications break. Both vehicles are equipped with the same highprecision IMU-based localization system, thus each can estimate accurately its own trajectory, as illustrated in Figure 8 (SV in blue, POV in red). The vehicles share their position via $\mathrm{V} 2 \mathrm{~V}$ communications. However, the $\mathrm{SV}$ is not able to estimate correctly the trajectory of the POV; the mapmatching algorithm running in SV localizes the POV on the wrong road (pink line in Figure 8). This was unexpected, as data used by SV and POV to localize the POV is the same. The main difference resides in the information received by the SV about the position of POV via V2V communications. It was observed that the link is not ideal. When the vehicles are far some messages were lost. The lack of positioning data, coupled with inaccuracies in the digital map geometric descriptions, plus the likely weakness in the map-matching function, leads to a large initial error in the trajectory estimation. The POV as seen from the SV is assigned to the wrong road with the error being propagated through the rest of the experiment. The position of the vehicles will be 
interpreted as not converging to the intersection. Vehicle localization is a major issue for situational awareness.

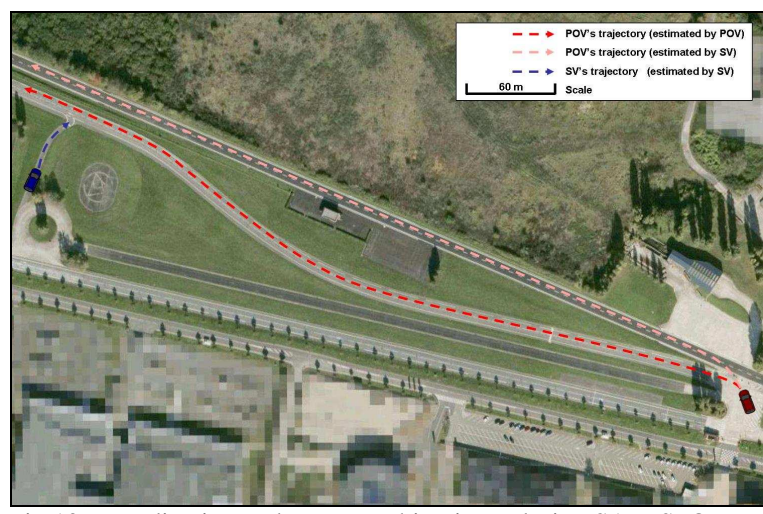

Fig 10. Localization and map-matching issue during SAFESPOT experiments

The third observation concerns time stamping and synchronization of the onboard computer clocks. Whilst most data will be time stamped using GPS time, once this is processed in different computers, the new time stamp will be associated to the processed data. The current implementation relies on the use of a NTP server to synchronize the computer clocks. When experiments were extended to three vehicles, this phenomenon was very noticeable.

The intersection applications have allowed for the identification of limits in the SAFESPOT framework. It has showed the potential of cooperative vehicles, the need for a structured digital world representation and precise low latency localization information.

\section{CONCLUSION}

Road intersections represent a high percentage of accidents and a source of stress to drivers. In this paper, the approach taken for the design of cooperative intersection systems was based on accidentology data was presented. The software components were developed within the SAFESPOT project. Passenger vehicles were used to demonstrate how vehicle-state variables could be exchanged to enhance regions of situational awareness, at speeds comparable to those found in real traffic conditions. The developments were software based implemented on COTS equipment. This spatio-temporal problem was constrained by the use of the Local Dynamic Map to represent the world where all applications infer their data.

Experiments showed the limitations of the wireless networks, the need for clock synchronisation and time stamping, and for means to compensate for communication delays or breaks as well as for data time alignment. The importance of localisation systems in cooperative vehicles was highlighted. Contrary to applications developed elsewhere, there was no reliance on road side units that could improve communications links. The testing of these systems remains a challenge in terms of resources, other than the test site, drivers, etc. means to quantify and log results are needed. Further, to trigger safety mechanisms, hazardous situations must be created, this implies a level of risk limiting evaluations. Large scale tests must take into account these considerations, and likely a combined strategy is needed, trials in dedicated sites and standard traffic conditions as well as advanced simulation.

Future work will centre on the building of a digital representation for decision making and on the development of metrics to effect quantitative performance assessments.

\section{ACKNOWLEDGMENT}

This work was co-funded by the European Commission as part of the SAFESPOT (Cooperative Systems for Road Safety) integrated project (http://www.safespoteu.org/ ). The authors would like to thank all the project partners in particular colleagues at Continental that collaborated with us on the implementation of the Intersections Use Cases.

\section{REFERENCES}

[1] European Road Safety Observatory, "Traffic Safety Basic Facts 2006 Junctions", SafetyNet, Project co-financed by the European Commission, January 2007.

[2] Source: CARE, IRF, IRTAD, TRACE, and National Statistics Databanks. Available: http://ec.europa.eu/transport/road_safety/specialist/statistics/care rep orts_graphics/index_en.htm,

[3] Europa, Communiqués de Presse RAPID, "Cars that talk: Commission earmarks single radio frequency for road safety and traffic management", IP/08/1240, Brussels, 5 August 2008. A available: http://europa.eu/rapid/pressReleasesAction.do?reference=IP/08/1240 \&format=HTML\&aged $=0$ \&language $=$ EN\& guiLanguage $=\mathrm{fr}$

[4] Cooperative Systems for Road Safety, SAFESPOT. Available: http://www.safespot-eu.org/

[5] Public Deliverables, SAFESPOT. Available: http://www.safespoteu.org/deliverables.html

[6] U.S. Department of Transportation National Highway Traffic Safety Administration, "Intersection Collision Avoidance Using ITS Countermeasures", Final Report: Performance Guidelines, September 2000, USA

[7] Ibanez-Guzman, J., Chapter, XI: The Localisation Problem in Cooperative Vehicle Applications, in Automotive Informatics and Communicative Systems: Principles in Vehicular Networks and Data Exchange, Edited by Guo, H., publishers Information Science Reference, April 2009

[8] Madhavan R., Messina E.R. \& Albus J.S., "Intelligent Vehicle Systems: A 4D/RCS Approach” New York, Nova Science Publishers, 2007

[9] Bartels, C., "SAFESPOT Local Dynamic Maps - Virtual world for safety applications", in Proceedings ITS World Congress, Beijing, 2007

[10] Car 2 Car Communications Consortium. Available: http://www.carto-car.org/index.php?id=1 accessed on March 2010

[11] Le, L., Festag, A., Baldessari, R. and Zhang, W., "V2X communication and intersection safety", in Advanced Microsystems for Automotive Applications, Springer, 2009, pp. 97-107

[12] Salim, F. D., "A context-aware framework for intersection collision avoidance", PhD thesis, , Monash University, Australia, 2008 